Macedonian Pharmaceutical Bulletin, 66 (Suppl 1) 71 - 72 (2020)

Online ISSN 1857 - 8969

UDC: 615.065:316.77]:340.13

DOI: 10.33320/maced.pharm.bull.2020.66.03.035

Short communication

\title{
Legal framework for use of social media in pharmacovigilance
}

\author{
Brankica Moskova $^{1}$, Ankica Gestakovska ${ }^{2}$, Zoran Sterjev $^{3}$, Aleksandra Grozdanova ${ }^{3}$, \\ Ljubica Suturkova $^{3}$, Aleksandra Kapedanovska Nestorovska ${ }^{3}$, Zorica Naumovska ${ }^{3}$ \\ ${ }^{1}$ Drug Safety Analyst IQVIA project member, 11 Oktomvri, 7500 Prilep, N. Macedonia \\ ${ }^{2}$ Quality Control Department, Replek Farm, Kozle 188, 1000 Skopje, N. Macedonia \\ ${ }^{3}$ Faculty of Pharmacy, Ss. Cyril and Methodius University, Mother Theresa 47, 1000 Skopje, N. Macedonia
}

\section{Introduction}

In accordance with the ICH E2D Guidelines, marketing authorization holders (MAH) are obliged to facilitate the collection of data on potential reports of suspected adverse reactions through their websites (for ex by providing direct reporting templates or through tools that enable direct communication). If MAH receives information about a suspected adverse reaction originating from a digital media sponsored by other companies, they should individually assess whether that report is appropriate to be submitted as an Individual Case Safety ReportISCR). Suspected adverse reactions received through websites or digital media sites are considered as spontaneous reports (Regulation (EU) No 520/2012).

Many companies on their own social media sites (Twitter, LinkedIn, etc.) to disseminate information about the company's products. In modern society social media could be used as potential tool in postmarketing drug safety monitoring and this approach is slowly but surely accepted by the pharmaceutical industry. For example, some companies use social networking sites to monitor health forums and share information between patients and healthcare professionals about specific pharmaceuticals or health conditions.

\section{Materials and methods}

Relevant European legislation was reviewed, in particular, Regulation (EU) No 520/2012, ICH E2D harmonized tripartite, EU (The Innovative Medicines Initiative Web-Recognizing Adverse Drug Reactions- IMI WEB-RADR) project enrolled in 182 countries, as well as PubMed, Medline and other relevant web sites for articles with empirical analysis, evaluating the impact of European and nonEuropean regulatory activities (Brosch et al., 2019; Ghosh et al., 2019; Regulation (EU) No 520/2012).

\section{Results and discussion}

The modern approach to tracking adverse drug reactions employs the Natural Language Program (NLP), which uses data for medicines and associated adverse reactions located on social media. Information posted on social media by drug users contains information on treatment outcomes and provides information that can be used to create reports of adverse reactions. These information are available although no valid reports for adverse drug reactions have been made and have crucial importance for all healthcare professionals and the pharmaceutical industry (Duh et al., 2017).

The type and volume of information for adverse drug reactions made available through the social

\footnotetext{
* brankica.moskova1977@gmail.com
} 
media to the pharmaceutical/healthcare industry cannot be easily provided in any other way. These include adverse reactions occurring in specific patient populations such as patients with rare diseases, pregnant women, nursing mothers, geriatric and pediatric populations or patients with comorbidities, which are usually excluded from clinical trials. There are still many questions that need to be answered concerning the ability of pharmaceutical companies to implement social media screening systems. One possibility for pharmaceutical companies is to establish patient support groups through social networking platforms to provide and share all relevant information about their products. These platforms can also carry out a series of activities that are part of risk minimization measures that sometimes only apply to healthcare professionals, but sometimes include patients as well. New tools and data sources, such as social media, provide numerous opportunities for enhancing public health, including opportunities to collect safety information, helping to generate new insights into drug benefit/risk profile, as well as unique insights into pharmacovigilance system development, in general. It is crucial that these opportunities are utilized ethically and in accordance with accepted regulations, in order to respect the privacy of data and to use it responsibly (Limaye and Saraogi, 2018). All healthcare professionals (doctors, nurses, dentists, and pharmacists) play pivotal role in prescribing, dispensing and administering of the drugs, but they also have an essential role in monitoring of treatment outcome, identifying and reporting of adverse events in postmarketing period, providing an efficient pharmacovigilance system. Social media can afford a serious platform for enhancing the connection between healthcare workers and their patients, providing a basis for sharing credible information about medications and therapeutic opportunities. Patients often engage in discussions with healthcare professionals on these sites and share their personal experiences on a specific drug, which can be a good basis for timely detection of new potential drug safety signals resulting in taking suitable actions for enabling efficient and safe use of the medicines available on the market (Caster et al., 2018).

There are few limitations in implementation in everyday pharmacovigilance practice. Medicines on social networks can be listed in a non-standard way, under their trademarks, generic names, or the active ingredients they contain.
The listed adverse events may be represented by idiomatic expressions that do not exist in medical lexicons and dictionaries. Informal communication on social media is an additional obstacle as bad grammar, shortcuts or slang are often used.

\section{Conclusion}

After establishment of appropriate legal framework and program languages the social media can be successfully used as effective source of information in modern pharmacovigilance practice worldwide. The risk-based approach applied aims to make better use of these new tools and data sources. The objectives of the developed principles are to guarantee continuous monitoring of drug safety and timely identification of potential safety signals without overloading pharmacovigilance systems.

\section{References}

Brosch, S., de Ferran, A.M., Newbould, V., Farkas, D., Lengsavath, M., Tregunno, P., 2019. Establishing a framework for the use of social media in pharmacovigilance in Europe. Drug Saf..42(8), 921930.

Caster, O., Dietrich, J., Kürzinger, M.L., Lerch, M., Maskell, S., Norén, G.N., Tcherny-Lessenot, S., Vroman, B., Wisniewski, A., van Stekelenborg, J., 2018. Assessment of the utility of social media for broadranging statistical signal detection in pharmacovigilance: results from the WEB-RADR Project. Drug Saf. 41(12), 1355-1369.

Duh, M.S., Ellman, B., Van Audenrode, M., Greenberg, P., Cremieux, P., 2017. Learning from social media for adverse event reporting. Law 360, New York.

Ghosh, R., Lewis, D., 2015. Aims and approaches of WEB-RADR: a consortium ensuring reliable ADR reporting via mobile devices and new insights from social media. Expert Opin. Drug Saf. 14(12), 18451853

International Council for Harmonisation of Technical Requirements for Pharmaceuticals for Human Use (ICH) ICH E2D harmonised tripartite guideline: postapproval safety data management: definitions and standards for expedited reporting, 2003.

Limaye, N., Saraogi, A., 2018. How social media is transforming pharma and healthcare. Appl. Clin. Trials 27(2). 